\title{
Comparison of Outcome of Close Reduction Versus Open Reduction in Treatment of Comminuted Mandibular Fracture
}

Sajid Ali Majeedano ${ }^{1}$, Kashif Ali Channar ${ }^{2}$, Wajid Ali Rajper ${ }^{3}$, Munawar Din Larik ${ }^{4}$, Muhammad Hassan Khoso ${ }^{5}$, Faiz Muhammad Khoso ${ }^{6}$ and Salman Shams ${ }^{7 *}$

${ }^{1}$ Lecturer, Oral and Maxillofacial Surgery Department, Muhammad Dental College, Pakistan

${ }^{2}$ Associate Professor, Oral and Maxillofacial Surgery Department, Faculty of Dentistry, Liaquat University of Medical and Health Sciences, Pakistan

${ }^{3}$ Lecturer, Oral Medicine Department, Faculty of Dentistry, Muhammad Dental College, Pakistan

${ }^{4}$ Dental Surgeon, Shaheed Mohtarma Benazir Bhutto Institute of Trauma, Karachi Pakistan

${ }^{5}$ Lecturer, Science of Dental Material Department, Faculty of Dentistry, Dow University of Health Sciences, Pakistan

${ }^{6}$ Lecturer, Oral Medicine Department, Faculty of Dentistry, Muhammad Dental College, Mirpurkhas, Pakistan

${ }^{7}$ Senior Lecturer, Oral and Maxillofacial Surgery Department, Faculty of Dentistry, Liaquat University of Medical and Health Sciences, Pakistan

\section{ABSTRACT}

Objective: To assess the efficiency of open reduction \& internal fixation versus close reduction with maxillomandibular fixation for the treatment of Comminuted mandibular fractures.

Study Design and Duration: This comparative study was carried out at Oral \& Maxillofacial Surgery Department, Liaquat University of Medical \& Health Science Jamshoro/Hyderabad, from November 2017 to October 2018.

Material and Methods: Total 60 patients after diagnosis of Comminuted mandibular fracture with age range from 18 to $45 \mathrm{years}$ of either gender were included. All the patients were divided in two groups, Group A was treated with open reduction with internal fixation and Group B was treated with closed reduction with maxillomandibular fixation. Each patient had called for follow-up after $2^{\text {nd }}$ week, $4^{\text {th }}$ week, and $6^{\text {th }}$ week. On follow-up postoperative infection, malocclusion, non-union/malunion of fracture fragments, facial asymmetry, exposed plates and sequestration of devitalized bone were documented

Results: Total 60 patients were included and divided in 2 groups, in patients of group A mean age was found $32.22 \pm 5.12$ years and mean age of the patients of Group B was 30.34 \pm 4.55 years. Males were found in the majority i.e 51 out of 60 patients while female were 9. Open reduction with internal fixation showed significant better outcome as compared to close reduction in the terms of complications, $p$-value 0.03 .

Conclusion: It was concluded that the open reduction with internal fixation is most effective reliable management technique with very low rates of early and late complication for the treatment of Comminuted fractures of the mandible as compared to close reduction.

KEYWORDS: Comminuted; Mandibular fracture; Open reduction; Internal fixation; Close reduction

Quick Response Code:

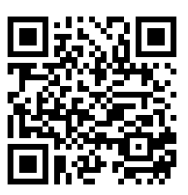

Address for correspondence: Salman Shams, Senior Lecturer, Oral and Maxillofacial Surgery Department, Faculty of Dentistry, Liaquat University of Medical and Health Sciences, Pakistan

Received: July 16, 2020

Published: July 28, 2020

How to cite this article: Sajid AM, Kashif AC, Wajid AR, Munawar D, Muhammad HK, Faiz MK, Salman S. Comparison of Outcome of Close Reduction Versus Open Reduction in Treatment of Comminuted Mandibular Fracture. 2020 - 2(4) OAJBS.ID.000199. DOI: 10.38125/OAJBS.000199 


\section{INTRODUCTION}

Comminuted fracture is defined as the presence of multiple fracture lines resulting in many small pieces within the same area of the mandible [1]. In extensive Comminuted fractures, multiple sites (exceeding one region and involving the neighboring region) of the mandible are splintered, crushed, pulverized, or broken into several pieces [1]. Extensive Comminuted mandibular fractures occur when a high-energy impact is applied onto a region of the mandible $[1,2]$. The high-energy impact seen in gun shots, high velocity traffic accidents, and falls from altitude can lead to enough concentrated force on the mandible to cause multiple sites of Comminuted fractures [1,2].

Comminuted mandibular fractures are serious injuries in the maxillofacial area. These fractures would affect the occlusion of the mandible and the appearance of lower regions of the face, which challenge the maxillofacial surgeons $[2,3]$. The treatment of these fractures necessitates the accurate anatomical reduction and stable fixation of the bony fragments, and to avoid post-operative complications like infection, malocclusion and disturbed facial appearance [3].

The predominantly males are affected more than females, and age between 16 to 30 years are mostly victimized. Most series of mandibular fractures report $5 \%$ to $7 \%$ as being Comminuted [4]. Comminuted mandibular fractures have been treated by a series of methods, including closed reduction, external pin fixation, internal fixation with Kirschner wires, and, open reduction with internal fixation (ORIF), using miniplates, compression plates, decompression plates, reconstruction plates [5].

In past, due to plate biocompatibility issue and to minimize the periosteal stripping, close reduction was the preferred method as compared recent literature which supports Open reduction \& internal fixation. Moreover, open reduction and internal fixation has been advocated for Comminuted fractures [6]. With the availability of Titanium implants for open reduction and internal fixation, it is observed that open reduction and internal fixation provides significant post-operative results, in term of facial symmetry and function [7]. Open reduction and internal fixation of these Comminuted mandible fractures with load bearing osteosynthesis allows for rapid healing and reduces the risk of non-union and malunion, immediate or early mandibular mobility, with good functional and aesthetic results $[8,9]$.

The main object was to premorbid dental occlusion with the appropriate bone healing and normal mouth opening and to decrease the morbidity with cost effective treatments.

\section{MATERIAL AND METHODS}

Study Design: Comparative study.

Sample Technique: Purposive, non- probability sampling / random numbering.

Sample Size: A sample size of 60 patients as calculated by confidence interval of $95 \%$.

GROUP A: Open reduction and internal fixation (reconstruction plates or/and miniplates) $=30$ patients

GROUP B: Close reduction with maxillomandibular fixation = 30 patients

\section{Inclusion Criteria:}

a) Patient age 18 to $45 y e a r s$.

b) Either gender.

c) Patient having comminuted fracture in mandible

d) Patient willing to participate in this study.

\section{Exclusion Criteria:}

a) Individuals below 18 years of age

b) Patient with a known systemic or bone diseases

c) Patient with missing mandibular fracture segment

d) Patient with malunion or non-union fracture

e) Patient with old fracture (more than 1 month)

\section{DATA COLLECTION PROCEDURE}

Patients meeting the inclusion criteria coming through Outpatient Department (OPD) or through Emergency Department were included in this study. A written consent was taken from every patient / attendant. Study was conducted after approval from Research Ethics Committee and member of REC of University.

Diagnosis of Comminuted mandibular fractures were done based on clinical examination, Orthopantomography (OPG) and posterior-anterior view of face. The patients were admitted in the hospital and were keep on nil per orally (NPO) before surgery for 6 hours. On the day of surgery, the general anesthesia consent was taken by hospital staff separately and the patients were prepared for general anesthesia. The standard and universal protocol for draping and preparation was done before starting surgery. Firstly, local anesthesia Xylocaine with $2 \%$ adrenaline 1:100,000 (Medicaine cartridges Company, Made in Korea) was given in the fracture region and then incision was given by the sterile surgical carbon steel blade \# 15 of carbon steel (Feather safety razor company Limited, Made in Korea) to access the fracture area. The reduction of fracture was done with the specific instrument and the fixation was achieved with reconstruction plates or/ and miniplates (Moin International, Pakistan) at fractured bone. Afterwards, wound were washed out with normal saline (Ostaka company, Made in Pakistan) for decontamination and incision was closed in two layers technique via sterile surgical suture (Vicryl 3-0, John \& Johnsons Company, Made in USA) and (Prolene 4-0, John \& Johnsons Company, Made in USA) surgical suture. The patient's attendant was advised to keep patient NPO for next 6 hours. The standard antibiotic with analgesics were given parentally for 2 days. The patients were discharge from the hospital after two days. Patients were prescribed antibiotics and analgesic orally for 5 days further. Each patient had called for follow-up after $2^{\text {nd }}$ week, $4^{\text {th }}$ week and $6^{\text {th }}$ week. On follow-up postoperative infection, malocclusion, nonunion/malunion of fracture fragments, facial asymmetry, exposed plates and sequestration of devitalized bone were documented.

In patients where closed reduction was carried out, Local anesthesia, Xylocaine with adrenaline 1:100000 (Medicaine company, made in Korea) was given with $1.8 \mathrm{ml}$ cartridges in the fracture region. After that, the procedure began by measuring the length of the arch bar with hook (Erich-type company, Made in Germany) for the mandibular and maxillary arches. The arch length ideally was placed from first molar to first molar in either jaws but may extend based on fracture locations. The arch bar was secured with stainless steel wires ( 24 or 26 gauge, Made in Pakistan) to the 
maxillary and mandibular facial/buccal cervical levels of the teeth When arch bar is secured, crossed intermaxillary wires were used to obtain pre-injury occlusion and fixation. The patient's mouth was closed for 4-6 weeks. The standard antibiotics with analgesic in syrup form was prescribed for 5 days. The patients were advised to take only liquid diet.

Each patient was called for follow-up after $2^{\text {nd }}$ week, $4^{\text {th }}$ week, and $6^{\text {th }}$ week. On follow-up the postoperative infection, malocclusion, non-union/malunion of fracture fragments, facial asymmetry, exposed plates and sequestration of devitalized bone were documented. On the sixth week follow up, the maxillomandibular fixation was removed. Data was analyzed by statistical software package SPSS version 20.0 .

\section{RESULTS}

In this study 60 patients were included and divided in 2 groups, in patients of group A mean age was found $32.22 \pm 5.12$ years and mean age of the patients of group B was $30.34 \pm 4.55$ years (Table 1 ).

Table 1: Age comparison in both groups; $n=60$.

\begin{tabular}{|c|c|c|}
\hline Age & Mean \pm SD. & P-value \\
\hline Group-A $\mathrm{n}=30$ & $32.22 \pm 5.12$ years & \multirow{2}{*}{0.09} \\
\hline Group-B $\mathrm{n}=30$ & $30.34 \pm 4.55$ years & \\
\hline
\end{tabular}

In this study male were found in the majority i.e 51 out of 60 patients, while female was 9 out of 10 , and no significant difference was found according to gender in both groups $p$-value 0.08 (Figure 1).

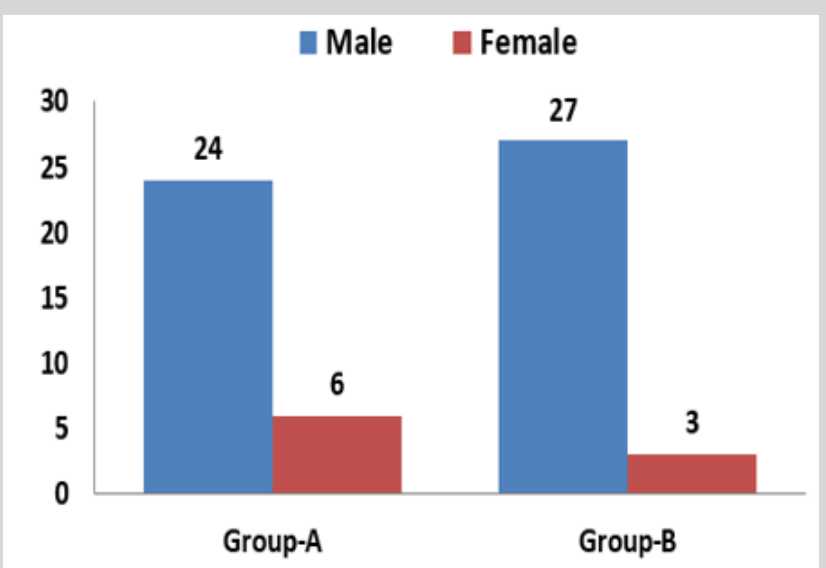

Figure 1: Distribution of cases according to gender $n=60$.

In this study most common cause of the injury was road traffic accident $50.0 \%$, followed by assault, gunshot and sports injury with percentage of $25.0 \%, 21.66 \%$ and $3.34 \%$ respectively (Figure 2).

According to the anatomical location of fractures, mostly were symphysis and Para symphysis $28.33 \%$ and $33.33 \%$ respectively, following by body of mandible was in $25 \%$ and angle of mandible was fractured in $13.34 \%$ of the patients (Table 2). In our study open reduction with internal fixation showed significant better outcome as compare to close reduction in the term of complications (early and late). $p$-value 0.03 (Table 3).

\section{DISCUSSION}

Total 60 patients were included and divided in 2 groups, mostly males were found with comminuted fracture of mandible and in patients of group A mean age was found32.22 \pm 5.12 years, and mean age of the patients of group B was $30.34 \pm 4.55$ years. As compare to our findings Amin et al. [10] reported that mean age of $32.7 \pm 12.83$ years. Similarly in the study of Neto et al. [11] it is stated that young males aged between 21 to 30 years old are mostly involved in these fractures, since this age group participates in the most dangerous exercises and sports, drives less carefully in our part of world, and are more prone to engage in episodes of interpersonal violence. However, many other studies reported that there is a worldwide trend to the increase of incidence in women, who are increasingly more exposed to the risk factors of this type of trauma $[12,13]$.

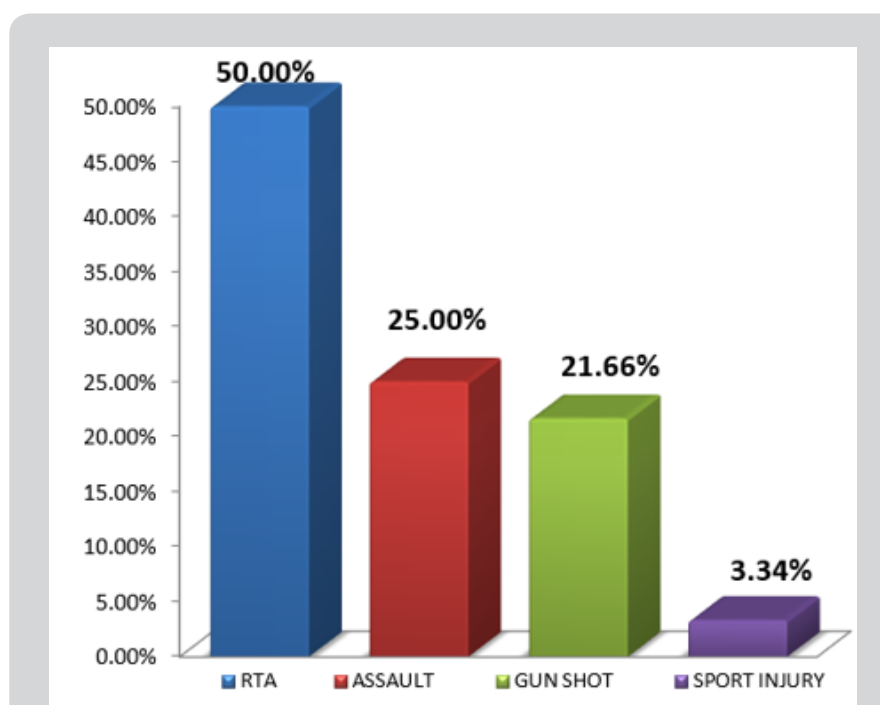

Figure 2: Cause of injury in the patients $n=60$.

Table 2: Site of the fractures; $n=60$.

\begin{tabular}{|c|c|c|}
\hline Site of fracture & Frequency & Percentage \\
\hline Symphysis & 17 & $28.33 \%$ \\
\hline Para symphysis & 20 & $33.33 \%$ \\
\hline Body of mandible & 15 & $25.00 \%$ \\
\hline Angle of mandible & 8 & $13.34 \%$ \\
\hline
\end{tabular}

Table 3: Postoperative complication assessment on followup; $n=60$.

\begin{tabular}{|c|c|c|c|}
\hline \multirow{2}{*}{ Follow-up } & \multicolumn{2}{|c|}{ Groups } & \multirow{2}{*}{$P$-value } \\
\hline & $\begin{array}{c}\text { Group-A } \\
n=\mathbf{3 0}\end{array}$ & $\begin{array}{c}\text { Group-B } \\
n=\mathbf{3 0}\end{array}$ & \\
\hline \multicolumn{3}{|l|}{ Early complications } & \multirow{4}{*}{0.05} \\
\hline Malocclusion & 2 & 5 & \\
\hline Facial asymmetry & 1 & 3 & \\
\hline Infection & 4 & 2 & \\
\hline \multicolumn{3}{|l|}{ Late complications } & \multirow{6}{*}{0.03} \\
\hline Infection & 1 & 3 & \\
\hline Expose plates & 1 & 2 & \\
\hline Facial asymmetry & 0 & 0 & \\
\hline Malocclusion & 0 & 0 & \\
\hline Nonunion & 0 & 2 & \\
\hline
\end{tabular}


In this study males were found in the majority 51 patients while female was 9 out of 60 . In the favor of this study Amin et al. [10] reported that out of 90 patients, there was a majority of male patients i.e. $84(93.3 \%)$ and $6(6.7 \%)$ were female with male to female ratio of $14: 1$. These fractures mostly caused by road traffic accident. In our study most common cause of the injury was road traffic accident $50.0 \%$, followed by assault, gun shot and sports injury with percentage of $25.0 \%, 21.66 \%$ and $3.34 \%$ respectively. On other hand Amin et al. [10] also found comparable findings and reported that the most common etiology of fracture mandible was road traffic accident (RTA), 57 (63.3\%) cases, followed by falls $14(15.6 \%)$, sports $9(10 \%)$, interpersonal violence $8(8.9 \%)$ and others $2(2.2 \%)$ which include gunshot wounds. Similarly many other studies also reported that theses fractures mostly caused by motor vehicle accidents, since it is a very resistant bone that requires a relatively strong trauma to have it fractured and may also be a result of practicing sports, injury caused by firearm or a weapon, physical assault, tooth extraction, workplace accidents, metabolic diseases and tumors [13-15].

In our study according to the anatomical location of fractures, mostly cases were of symphysis and parasymphysis 33.33\% and $28.33 \%$ respectively followed by body of mandible in $25 \%$ and angle of mandible fractured in $13.34 \%$ of the patients. On other hand in the studies of Miloro [16] and Soares [17] the main fractured sites have been reported as $16 \%$ to $33 \%$ involving the body, $23.1 \%$ to $27.3 \%$ the angle of mandible, 19.5 to $29.3 \%$, the condyle, $8.4 \%$ to $22 \%$, mandibular symphysis, $16 \%$ to $33 \%$ Para symphysis, $1.7 \%$ to $2.4 \%$, the ramus; $0.2 \%$ to $4.8 \%$ the coronoid process and $1.4 \%$ to $3.1 \%$ in the alveolar process. These findings slightly vary as compare to our study, and this may because the location and the pattern of mandibular fractures are determined by the mechanism of the injury and the direction of the force vector. In addition, other factors such as age of the patient, the presence or absence of teeth, the presence of pathologies and the physical properties of the etiologic agent also have a direct effect on the characteristics and results of the fracture [16].

In our study open reduction with internal fixation showed significant better outcome as compare to close reduction in the term of complications and non-union p-value 0.03. Similarly, Hirani et al. [18] demonstrated that the open reduction with internal fixation is better surgical option in symphysis and Para symphysis mandible fracture with shorter surgical time and well tolerated by patients. In another Pakistani study conducted by Ajmal et al. [19] reported that Open reduction and internal fixation with Miniplate has proven to be the most effective method of rigid fixation, associated with minimal morbidity and early mobilization and return to work. The authors observed the incidence of infection and malocclusion in $12.9 \%$ and $4.7 \%$ respectively. As well in this study malocclusion was found in 2 patients of group A and in 5 patients of group B, while infection was found in 4 patients of group $A$ and in 2 patients of group B. In the comparison of this study Richter et al. [20] reported that success of open reduction with internal fixation in Comminuted fractures of the mandible is directly related to two fundamental principles: fixation needs to support the full functional loads and absolute stability of the fracture. On the other hand, in previous studies [21,22] mention that the ideal treatment for Comminuted fractures of the mandible must be performed by means of open reduction with internal fixation with large reconstruction plates. They suggest that a small number of complications would occur if the absolute stability of this fracture were achieved. They point out the advantages of open reduction with internal fixation as being the anatomic reduction of the fractured fragments and early return of function. In favor of our study Smith [23] evaluated retrospectively the success rate of open reduction with internal fixation used to treat 16 consecutive Comminuted mandibular fracture.

\section{CONCLUSION}

It was concluded that the open reduction with internal fixation most effective reliable, management technique with very low rates of early and late complication for the treatment of Comminuted fractures of the mandible as compare to close reduction.

\section{REFERENCES}

1. Channar KA, Dal AQ, Safia, Warriach RA (2011) Comparison of open reduction and internal fixation versus closed reduction and maxillomandibular fixation for the treatment of gunshot injuries of mandible. JLUMHS 10: 168-173.

2. Lee K, Yoon K, Park KS (2014) Treatment of extensive comminuted mandibular fracture between both mandibular angles with bilateral condylar fractures using a reconstruction plate: a case report. J Korean Assoc Oral Maxillofac Surg 40: 135-139.

3. Li P, Tang W, Liao C (2015) Clinical evaluation of computer-assisted surgical technique in the treatment of comminuted mandibular fractures. J Oral Maxillofac Surg, Med and Patho 27: 332-336.

4. Alpert B, Tiwana PS, Kushner GM (2009) Management of comminuted fractures of the mandible. Oral Maxillofac Surg Clin N Am 21: 185-192.

5. Abreu MER, Viegas VN, Ibrahim D (2009) Treatment of comminuted mandibular fractures: A critical review. Med Oral Patol Oral Cir Bucal 14: $247-251$.

6. Dai J, Shen G, Yuan H (2015) Titanium mesh shaping and fixation for the treatment of comminuted mandibular fractures. J Oral Maxillofac Surg 1: $1-11$.

7. Futran ND (2008) Management of comminuted mandible fractures. Operat Tech in Otolaryngol 19: 113-116.

8. Ellis E, Muniz O, Anand K (2003) Treatment considerations for comminuted mandibular fractures. J Oral Maxillofac Surg 61: 861-870.

9. Rana MA, Warraich R, Rashad A (2014) Management of comminuted but continuous mandible defects after gunshot injuries. Int J Care Injure 45(1): 206-211.

10. Amin M, Babar A, Ibrahim MW, Awan MU (2016) Post-operative complications in comminuted mandibular fracture management; comparison of three different treatment modalities. Pakistan Armed Forces Medical Journal 66(5): 720-725.

11. Neto IC, Franco JM, Santana MD, Batista HM, Soares EC, et al. (2015) Simplification on the reduction of comminuted mandibular fractures for stable internal fixation. Int Arch Med 12(8): 1-9.

12. Motamedi MH, Dadgar E, Ebrahimi A, Shirani G, Haghighat A, et al. (2014) Pattern of maxillofacial fractures: a 5-year analysis of 8,818 patients. J Trauma Acute Care Surg 77(4): 630-634.

13. Gassner R, Rudisch A, Ulmer H (2003) Cranio-maxillofacial trauma: A 10year review of 9543 cases with 21067 injuries. J Craniomaxillofac Surg 31(1): 51-61.

14. Motamedi MH (2003) An assessment of maxillofacial fractures: a 5-year study of 237 patients. J oral Maxillofac Surg 61: 61-64.

15. Zhi LI, Zu-bing LI (2011) Clinical charcteristics and treatment of multiple site comminuted mandible fractures. J Craniomaxillofac Surg 39: 296299.

16. Miloro M (2011) Peterson's principals of oral and maxilla facial surgery. $2^{\text {nd }}($ edn.). Philadelphia, USA

17. Soares EC, Santana MD (2011) Clinical Charcteristics and treatment of multiple site comminuted mandible fractures. J Cranio-Maxillofac Surg 39: 276-282. 
18. Hirani NN, Pujara N (2015) Comparison of open reduction and interna fixation in case of symphysis and parasymphysis mandible fracture. International Journal of Science and Research (IJSR) 4(6): 2129-2131.

19. Ajmal S, Khan MA, Jadoon H, Malik SA (2007) Management protocol of mandibular fractures at Pakistan institute of medical sciences, Islamabad, Pakistan. J Ayub Med Coll Abbottabad 19: 226-234.

20. Scolozzi P, Richter M (2003) Treatment of severe mandibular fractures using AO reconstruction plates. J Oral Maxillofac Surg 61: 458-461.
21. Smith BR, Teenier TJ (1996) Treatment of comminuted mandibular fractures by open reduction and rigid internal fixation. J Oral Maxillofac Surg 54: 328-331.

22. Fonseca JR, Walker RV, Bettis JN, Dexter BH (1997) Oral and maxillofacial trauma volume 1, $2^{\text {nd }}$ (edn.). Saunders Company, Philadelphia, USA.

23. Smith BR, Johnson JV (1993) Rigid fixation of comminuted mandibular fractures. J Oral Maxillofac Surg 51: 1320-1326. 